\title{
Nichtlineare Rauschmodellierung von LC Tank VCOs
}

\author{
T. Thiessen, J.-K. Bremer, and W. Mathis
}

Leibniz Universität Hannover, Institut für Theoretische Elektrotechnik, Appelstr. 9A, 30167 Hannover, Germany

Zusammenfassung. Im Rahmen dieser Arbeit wird ein alternativer Ansatz zur Phasenrauschoptimierung von LC-Tank Oszillatoren (VCOs) unter Verwendung stochastischer Differentialgleichungen vorgestellt. Zunächst werden die linearen Ansätze von Leeson, Hajimiri und Lee analysiert und bewertet. Danach wird ein Konzept vorgestellt, mit dem man die Rauscheigenschaften von VCOs auf der Grundlage stochastischer Differentialgleichungen und Fokker-PlanckGleichungen untersuchen kann. Ziel dieser Arbeit ist eine Beschreibung des Phasenrauschens auf der Basis einer nichtlinearen Rauschmodellierung, welche Parameter eines VCOs fr eine Optimierung beinhaltet. Es wurde ein Matlab-Tool erstellt und die Funktionalitt anhand von Simulationen verifiziert.

\section{Einleitung}

Phasenrauschen ist in Kommunikationssystemen ein wichtiger Parameter beim Entwurf von VCOs. Das reziproke Mischen von Phasenrauschen und Trgersignal verursacht eine Vielzahl von unerwünschten Effekten wie Nachbarkanalstörungen, erhöhte Bitfehlerrate (BER) und Synchronisationsproblemen in digitalen Systemen. Mit Modulationsverfahren wie z.B. Orthogonal Frequency-Division Multiplex (OFDM), steigt auch die Nachfrage nach besseren spektralen Eigenschaften. Demzufolge ist eine Optimierung des Phasenrauschens und die Möglichkeit zur Modellierung von großer Bedeutung.

Oszillatoren sind elektronische Schaltungen mit periodischen Strömen und Spannungen die folgende Eigenschaften besitzen: (a) feste Frequenz (bzw. einstellbar) (b) feste Amplitude (bzw. einstellbar) (c) Amplitude unabhängig von den Anfangsbedingungen und (d) qualitatives Verhalten ändert

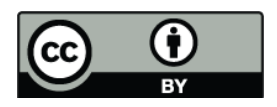

Correspondence to: J.-K. Bremer (bremer@tet.uni-hannover.de)

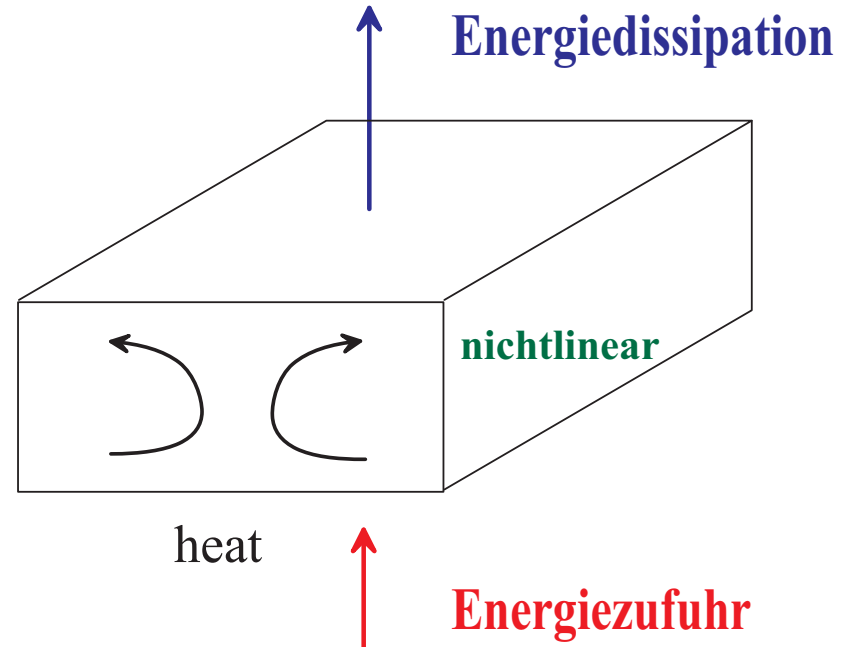

Abb. 1. Dissipation und Grenzzyklus.

sich nicht beim Hinzufügen von kleinen parasitären Störungen.

Daraus ergibt sich, dass ein Oszillator mit Hilfe eines nichtlinearen Systems modelliert werden muss, welches asymptotisch stabile periodische Lösungen aufweist (vgl. Mathis und Russer, 2005; Kurz und Mathis, 1994). Im Rahmen der mathematischen Theorie dynamischer Systeme werden solche Lösungen durch stabile Grenzzyklen beschrieben, bei denen es sich um isolierte geschlossene Kurven im Zustandsraum handelt. Alle transienten Lösungen mit Anfangsbedingungen, die nicht auf der Kurve starten, laufen asymptotisch in diese Kurve ein (Jordan und Smith, 1987). Um dieses Verhalten zu gewährleisten, muss das nichtlineare System kontinuierlich Energie aufnehmen und abgeben (vgl. Abb. 1). Nach dem Jouleschen Gesetz sind Ohmsche Widerstände

Published by Copernicus Publications on behalf of the URSI Landesausschuss in der Bundesrepublik Deutschland e.V. 


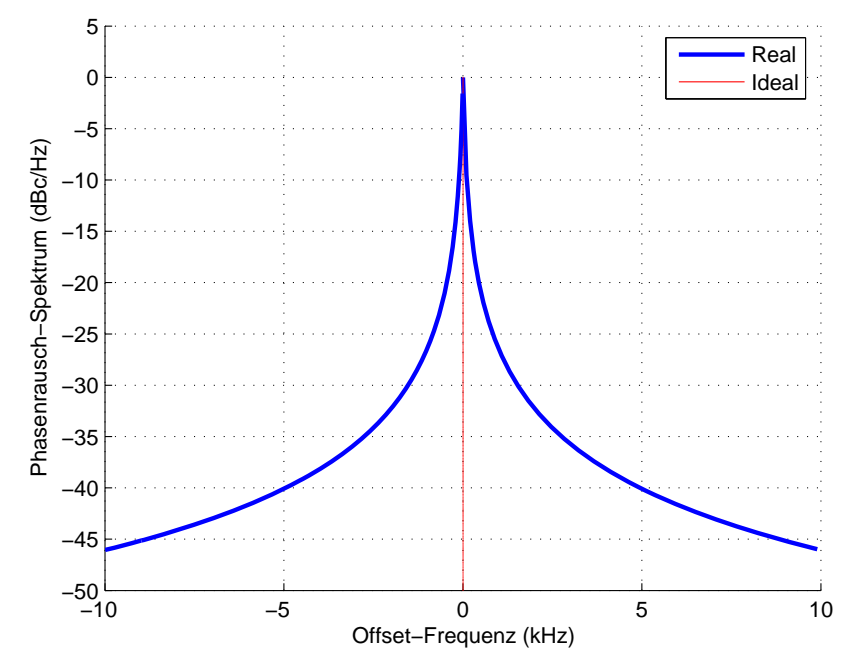

Abb. 2. Phasenrauschspektrum eines Oszillators.

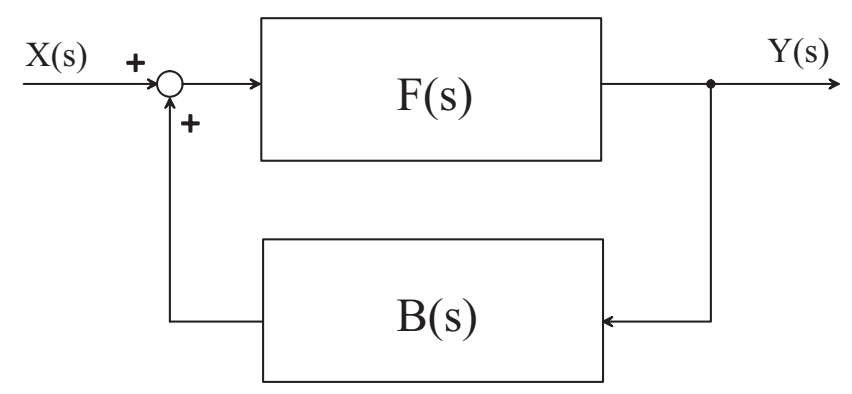

Abb. 3. Blockschaltbild eines positiven Feedback Regelkreises.

an ein Wärmebad gekoppelt. Durch diese Kopplung geht Energie aus einer Oszillatorschaltung in das Wärmebad über. Die Erhöhung der kinetischen Energie im Wärmebad führt zu einer Rückwirkung auf das System und verstärkt eine Teilchen-Fluktuation im Widerstand. Dieser Vorgang wird durch das Dissipations-Fluktuationstheorem beschrieben (Van Kampen, 1992). Oszillatoren sind folglich keine deterministischen Systeme, was eine nichtlineare Rauschmodellierung zur Beschreibung von Oszillatoreigenschaften erfordert.

Das Rauschen in Oszillatoren tritt als Amplituden- und Phasenrauschen in Erscheinung, die i.a. gekoppelt sind. Eine vollständige Entkopplung der Rauscheffekte kann dazu führen, dass einige Effekte unberücksichtigt bleiben (z.B. Frequenzshift, siehe Maffezoni, 2007; Hänggi und Riseborough, 1983). Mit Hilfe der Mittelungsmethode nach Bogoliubov (1963) und Stratonovich (1967) kann man aus den gekoppelten Amplituden- und Phasengleichungen eine Näherungsbeschreibung bestimmen, mit denen man auch die eben erwähnten Effekte analysieren kann.

Die periodischen Lösungen von Oszillatoren liefern ein Linienspektrum (vgl. Abb. 2).

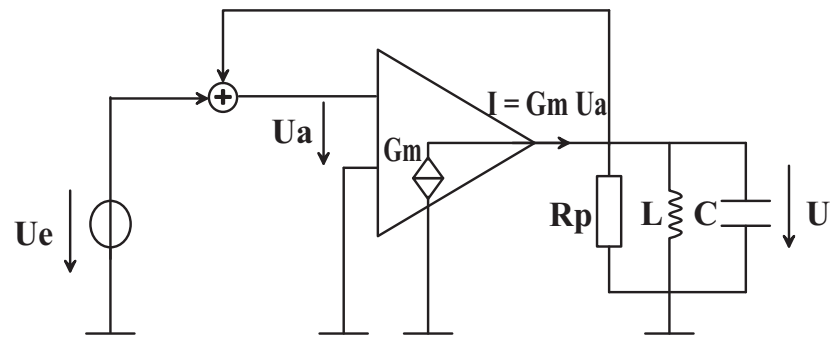

Abb. 4. Blockschaltbild eines positiven LC-Feedback Oszillator.

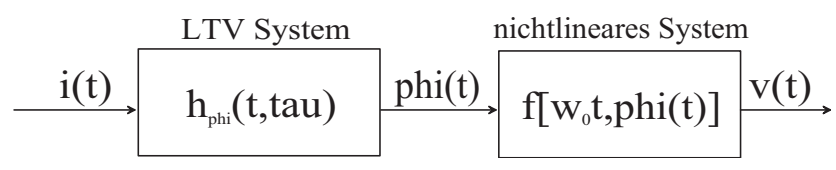

Abb. 5. Übertragungssytem nach Hajimiri und Lee.

Basierend auf einer Arbeit von Lax (1967) wird im Folgenden gezeigt, dass die Linienaufweitung der spektralen Leistungsdichte durch das Einbringen einer Rauschquelle in die nichtlineare Differentialgleichung des Oszillators erklärbar ist und nicht durch eine Filterung, wie in linearen Rauschmodellen, erzeugt wird. Weiterhin wird eine nichtlineare Rauschmodellierung von Oszillatoren vorgestellt und deren Ergebnisse anhand eines Van der Pol Oszillators exemplarisch dargestellt. Mit Hilfe einer in Matlab implementierten Toolbox können die Ergebnisse der numerischen Simulation präsentiert werden.

\section{Lineare Modelle}

In vielen Untersuchungen (Leeson, 1966; Craninckx und Steyaert, 1995; Sauvage, 1977; Nallatamby et al., 2003) zum Phasenrauschen werden linear zeitinvariante (LTI) Modelle für High-Q und Quarz-Oszillatoren verwendet. Die bekannte Leeson Formel (Leeson, 1966) kann unter Berücksichtigung des Barkhausen-Kriteriums bestimmt werden. Dazu wird das Rückkopplungsnetzwerk aus Abb. 3 mit Hilfe eines äquivalenten Netzwerkmodells (siehe Abb. 4) realisiert und näherungsweise die Impedanz

$$
Z\left(\omega_{0}+\Delta \omega\right) \approx \frac{R_{p}}{1+j 2 Q_{L} \frac{\Delta \omega}{\omega_{0}}}
$$

des Parallelschwingkreises bei der Frequenz $\omega_{0}+\Delta \omega$ bestimmt $\left(Q_{L}\right.$ : Schwingkreisgüte). Durch Aufstellen der Übertragungsfunktion $H(j \omega)$

$H(j \omega)=\frac{U_{a}}{U_{e}}=1+\frac{1}{j 2 Q_{L} \frac{\Delta \omega}{\omega_{0}}}$

und Annahme eines Eingangsrauschspektrum der Form

$S_{x}(\Delta \omega)=2 F k T R \cdot\left(1+\frac{\omega_{c}}{\Delta \omega}\right)$, 
wobei $F$ die Effektive Rauschzahl, $R$ der rauschende Widerstand und $\omega_{c}$ die Eckfrequenz des $1 / f$-Rauschens ist, kann das Ausgangsphasenrauschen mit Hilfe der WienerLee-Beziehung

$L(\Delta \omega)=S_{x}(\Delta \omega) \cdot|H(j \omega)|^{2}$

ermittelt werden $\mathrm{zu}$

$L(\Delta \omega)=2 F k T R \cdot\left[1+\left(\frac{\omega_{0}}{2 Q_{L} \Delta \omega}\right)^{2}\right] \cdot\left(1+\frac{\omega_{c}}{\Delta \omega}\right)$.

Die Faktoren $F$ und $\omega_{c}$ stellen hierbei die empirisch zu bestimmenden Parameter dar. Trotz großer praktischer Bedeutung dieser Modelle, basieren sie auf einer starken Vereinfachung des Problems. Sie verdecken auch die fundamentale Fragestellungen, wie die, dass rauschende Oszillatoren eine spektrale Dispersion aufweisen, die nicht mit einem linearen Modell erklrt werden kann (Demir et al., 2000).

Um die Nichtlinearitäten in High-Q Oszillatoren zu berücksichtigen und die Rauschanalyse zu verbessern, wurden einige Untersuchungen mittels linear zeitvarianter (LTV) Methoden durchgeführt (vgl. Hajimiri und Lee, 1998). Das Verfahren von Hajimiri und Lee basiert auf einem zweistufigen Übertragungssystems (vgl. Abb. 5), welches den Rauschstrom in die Ausgangsspannung transformiert. Das erste, LTV System repräsentiert die Rauschstrom-PhasenUmsetzung durch die von Hajimiri und Lee definierte, zeitvariante "Impulse Sensitivity Function" (ISF). Das zweite, nichtlineare System stellt eine Phasenmodulation dar, welche die Phasenänderung in die Ausgangsspannung transformiert. Basierend auf deterministischen Störungen, liefern LTV Analysen eine bessere Abschätzung des Spektrums als LTI Verfahren (Demir et al., 2000). Dennoch bleiben bei der LTV Analyse mit stochastischen Störungen einige nichtphysikalische Ergebnisse der LTI Analyse, wie das unendlich groe Rauschen bei der Oszillationsfrequenz, bestehen und ermöglichen wenig Einblick in die grundlegenden physikalischen Mechanismen des Phasenrauschens.

\section{Stochastische Beschreibung von VCOs}

Um die tatsächlichen physikalischen Rauschvorgänge in VOCs zu modellieren, gehen wir zunächst von einer deterministischen Beschreibung solcher Schaltungen aus, die das nichtlineare Verhalten und insbesondere das Auftreten von Grenzzyklen repräsentieren. Allgemeine nichtlineare Schaltungen lassen sich in folgender Weise modellieren

$\frac{d \mathbf{x}}{d t}=\mathbf{a}(\mathbf{x}(t), t)$.

Das thermische Rauschen in einer Schaltung wird nun durch einen nichtlinear angekoppelten weißen VektorRauschprozess $\xi(t)$ modelliert, d.h. es ergibt sich

$\frac{d \mathbf{x}}{d t}=\mathbf{a}(\mathbf{x}(t), t)+\mathbf{B}(\mathbf{x}(t), t) \xi(t)$, wobei $\xi(t)$ ein weißer Rauschprozess ist, der folgende Eigenschaften besitzt $(\langle\cdot\rangle$ ist der Mittelwertoperator)

$$
\left\langle\xi_{i}(t)\right\rangle=0, \quad\left\langle\xi_{i}(t) \xi_{j}(t+\tau)\right\rangle=\delta_{i j} \delta(\tau)
$$

und die Anfangsbedingungen $\mathbf{x}\left(t_{0}\right)=\mathbf{x}_{0}$ mit $i, j=1, \cdots, n$ vorgegeben sind. Differentialgleichungen dieses Typs gehören zur Klasse stochastischer Differentialgleichungen (SDEs). Unsynchronisierte Oszillatorschaltungen besitzen keine explizite Zeitabhängigkeit, so dass für den Driftvektor $\mathbf{a}=\mathbf{a}(\mathbf{x}(t))$ und für die Diffusionsmatrix $\mathbf{B}=\mathbf{B}(\mathbf{x}(t))$ gilt. Vom Standpunkt der statistischen Thermodynamik ist ein Grenzzyklus (vgl. Abb. 1) ein dynamischer Prozess, der fernab vom Gleichgewicht stattfindet. Damit können die von Weiss und Mathis (Mathis und Weiss, 2003; Weiss und Mathis, 1999) entwickelten Methoden der Rauschbeschreibung in nichtlinearen Schaltungen nicht mehr angewendet werden, da sie sich auf Prozesse in der "Nähe" des Gleichgewichts beziehen.

Man kann zeigen, dass die Lösungen von SDEs stochastische Prozesse sind, die Eigenschaften eines Markow Prozesses besitzen. Solche Prozesse werden mit Hilfe der sogenannten Chapman-Kolmogorov-Integralgleichung (CKGleichung) charakterisiert; vgl. z.B. Van Kampen (1992). Löst man die CK-Gleichung mit Hilfe einer sogenannten Kramers-Moyal-Entwicklung, in der bis auf die ersten beiden Terme sämtliche Terme verschwinden, dann erhält man eine sogenannte Fokker-Planck-Differentialgleichung (FP-Gleichung)

$$
\begin{aligned}
\frac{\partial p\left(\mathbf{x}, t \mid \mathbf{x}_{0}, t_{0}\right)}{\partial t} & =-\sum_{i=1}^{n} \frac{\partial}{\partial x_{i}}\left[[\mathbf{a}]_{i} p\left(\mathbf{x}, t \mid \mathbf{x}_{0}, t_{0}\right)\right] \\
& +\frac{1}{2} \sum_{i, j=1}^{n} \frac{\partial^{2}}{\partial x_{i} \partial x_{j}}\left[\left[\mathbf{B B}^{\mathbf{T}}\right]_{i j} p\left(\mathbf{x}, t \mid \mathbf{x}_{0}, t_{0}\right)\right],
\end{aligned}
$$

wobei $p(\mathbf{x}, t)$ die Übergangswahrscheinlichkeit des zugehörigen Markow-Prozesses ist; siehe Risken (1989). Die Lösung der FP-Gleichung ist bei realen Schaltungen aufgrund der vielen Variablen und der Parabolizität der Gleichung sehr aufwendig. Exakte Lösungen sind mit Ausnahme der Van der Pol Gleichung kaum verfügbar (Risken, 1989) und auch die Numerik solcher Gleichungen ist schwierig. Deshalb verwenden wir im folgenden numerische Verfahren für die SDE (Gl. 6).

\section{Linienaufweitung der spektralen Leistungsdichte}

Bei der gewöhnlichen quasilinearen Betrachtungen von Rauschen in nichtlinearen Systemen, verursacht das Rauschen einen additiven Effekt zum Trägersignal. Wendet man die quasilinearen Verfahren bei einem Oszillator an, würde man ein Spektrum der Ausgangsspannung erhalten, das aus einem diskreten Spektrum fr den Grenzzyklus und einem additiven Rauschgrund besteht. Wie man jedoch experimentell zeigen 


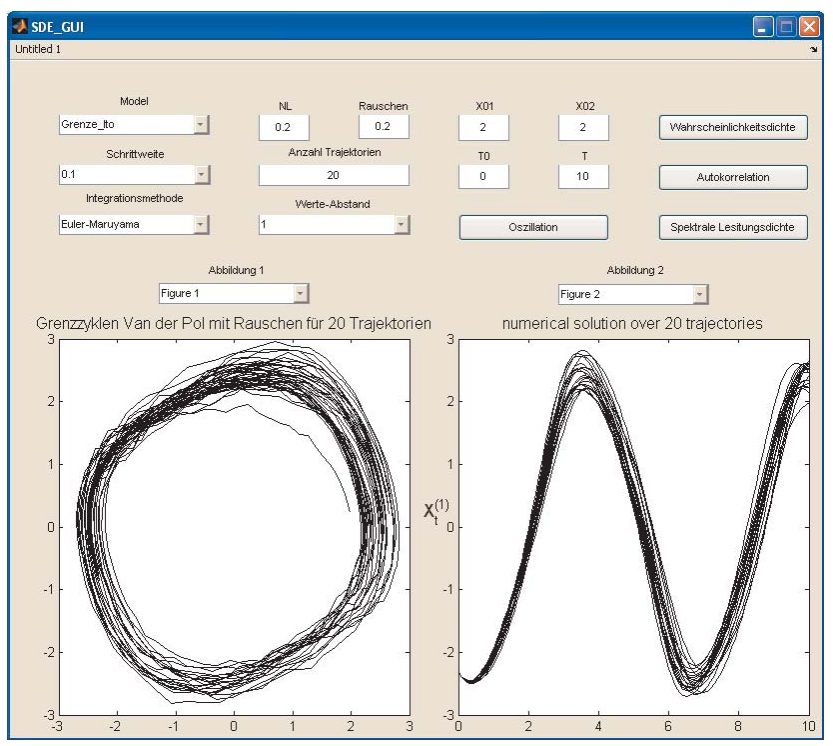

Abb. 6. Grafische Benutzeroberfläche.

kann (siehe Lax, 1967), erzeugt Rauschen in einem Oszillator eine Linienaufweitung des Spektrums, die man als Lorentzlinie bezeichnet.

Voraussetzung für eine quasilineare Betrachtung von nichtlinearen Systemen ist dessen Stabilität. Die Amplitude eines Grenzzyklus weist ein stabiles, die Phase jedoch ein instabiles Verhalten auf. Somit ist eine quasilineare Betrachtung der Phase nicht möglich.

Folgende Differentialgleichung für die Phase kann abgeleitet werden (Lax, 1967)

$\frac{d \phi}{d t}=\xi(t) \cdot \cos \left(\phi+\omega_{0} t\right)$.

Dabei beschreibt $\xi(t)$ eine weiße, Gaußsche Zufallsvariable, die proportional zum Eingangsrauschen ist. $\phi+\omega_{0} t$ ist die Momentan-Phase und $\omega_{0}$ die Oszillatorfrequenz. Gleichung (9) beschreibt einen nichtstationären Markow-Prozess dessen Diffusionskonstante zeitabhängig und proportional zu $\cos ^{2}\left(\omega_{0} t+\phi\right)$ ist. Für große Zeiten $\left(t \gg\left(\omega_{0}\right)^{-1}\right)$ kann jedoch eine FP-Gleichung abgeleitet werden (Risken, 1989). Man kann zeigen (Lax, 1967), dass zwischen der nichtlinearen SDE Gl. (9), dem vereinfachten, linearen Prozess

$d \phi / d t=\xi(t) \cdot \cos \left(\omega_{0} t\right)$

und dem reduzierten Prozess

$d \phi / d t=1 / \sqrt{2} \cdot \xi(t)$

kein Unterschied zwischen ihren statistischen Verhalten besteht. Der reduzierte Prozess $\phi$ entspricht einer gewöhnlichen Brownschen Bewegung (Wiener Prozess). Die spektrale Leistungsdichte (SLD) der Zustandsgröße

$\mathbf{x}(t)=\mathbf{a}(t) \cdot e^{\phi(t)} e^{-j \omega_{0} t}$ kann nun mit Hilfe der Fourier-Transformation der $\mathrm{Au}-$ tokorrelationsfunktion (AKF) bestimmt werden (WienerKhintchine Theorem).

$\left\langle\mathbf{x}^{*}(t) \mathbf{x}(0)\right\rangle \approx\left\langle|\mathbf{a}|^{2}\right\rangle\left\langle e^{j[\phi(t)-\phi(0)]}\right\rangle e^{j \omega_{0} t}$

Die Amplituden-Fluktuationen $a(t)$ wurden bei der Bestimmung der AKF in Gl. (13) vernachlässigt. Da $\phi$ eine mittelwertfreie Gauß-Variable ist, ergibt sich folgende Beziehung

$\left\langle e^{j[\phi(t)-\phi(0)]}\right\rangle=e^{-\frac{1}{2}\left\langle[\phi(t)-\phi(0)]^{2}\right\rangle}$.

Für eine weitere Betrachtung (für $t \gg\left(\omega_{0}\right)^{-1}$ ) kann der lineare Prozess $\phi$ verwendet werden, der ein Wiener Prozess ist und bei dem die mittlere quadratische Verschiebung linear mit der Zeit ansteigt (Papoulis, 1965)

$\left\langle[\phi(t)-\phi(0)]^{2}\right\rangle=W \cdot|t|$.

Folglich kann die AKF in die Form

$\left\langle\mathbf{x}^{*}(t) \mathbf{x}(0)\right\rangle=\left\langle|\mathbf{a}(t)|^{2}\right\rangle e^{-\Lambda_{p} \cdot|t|} \cdot e^{j \omega_{0} t}$

mit $\Lambda_{p}=\frac{1}{2} W$ umgeschrieben werden.

Das Rauschspektrum erhält somit die bekannte Lorentzform:

$$
\begin{aligned}
\left\langle\mathbf{x}_{\omega}^{*} \mathbf{x}_{\omega}\right\rangle & =\int_{-\infty}^{\infty} e^{-j \omega t} d t\left\langle\mathbf{a}^{*}(t) \mathbf{a}(0)\right\rangle \\
& =\left|\mathbf{a}_{0}\right|^{2} \frac{\Lambda_{p}}{\Lambda_{p}^{2}+\left(\omega-\omega_{0}\right)^{2}}
\end{aligned}
$$

Der Parameter

$\Lambda_{p}=\frac{1}{2} W=\frac{1}{4}\left(G^{2}\right)_{\omega_{0}}=\frac{1}{4} \frac{\left(e^{2}\right) \omega_{0}}{\left|L^{\prime} a_{0}\right|^{2}}$

gibt ein Maß für die Breite der SLD wieder, wobei $L^{\prime}$ eine Induktivität, $\left(e^{2}\right)_{\omega_{0}}$ die Leistung der Rauschquelle und $\left|\mathbf{a}_{0}\right|$ den Betrag der Amplitude bei der Oszillatorfrequenz $\omega_{0}$ widerspiegelt. Damit ist gezeigt, dass der Rauschprozess selbst eine Linienaufweitung der SLD in Form einer Lorentzlinie erzeugt. Eine entsprechende Beziehung der SLD fr einen LC-VCO wurde in der Arbeit [Magierowski und Zukotynski (2004)] vorgestellt, welche einen mathematischen $\mathrm{Zu}$ sammenhang zwischen Designparametern und SLD formuliert. In dieser Arbeit wird gezeigt, dass durch eine numerische Lösung der SDE die AKF und SLD, durch Bestimmung der einzelnen Trajektorien und anschlieender Mittelung, angenähert werden können (siehe Abschnitt 5).

\section{Simulationsergebnisse}

Die Van der Pol Gleichung reprsentiert eine Klasse von Differentialgleichungen mit Grenzzyklus, die zur Beschreibung von VCOs geeignet sind. Hier wird diese Gleichung zur Illustration der Simulationsmethoden verwendet:

$\ddot{x}+\varepsilon\left(x^{2}-1\right) \dot{x}+x=a \cdot \xi$ 


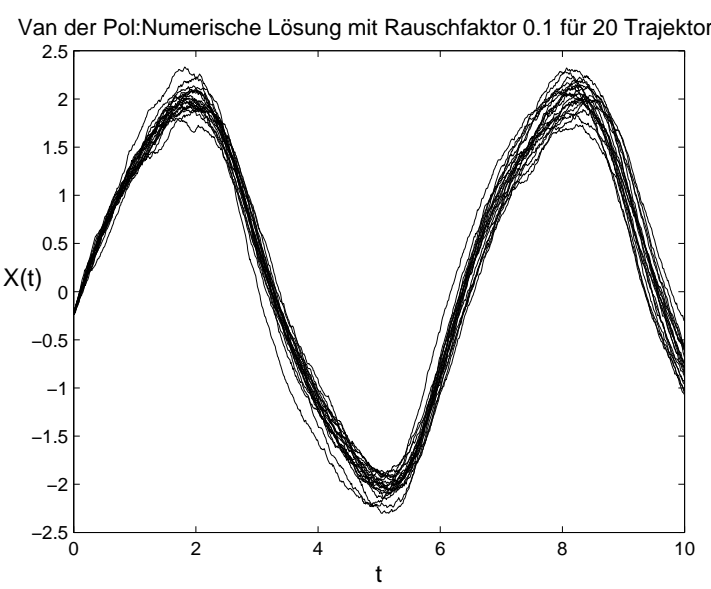

(a)

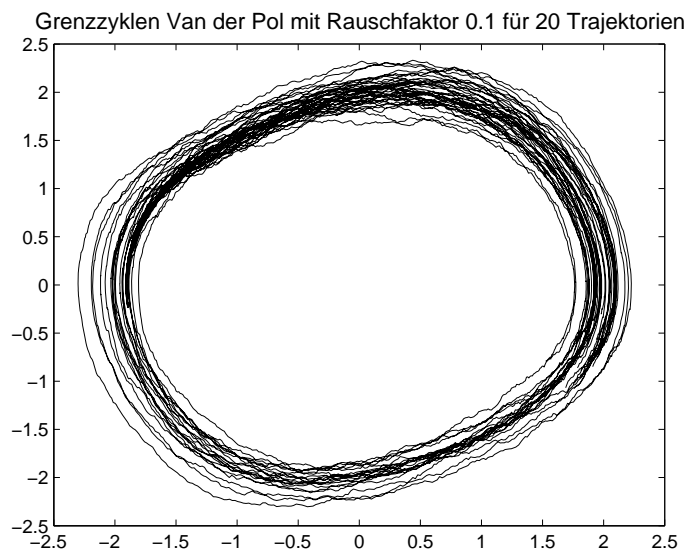

(c)

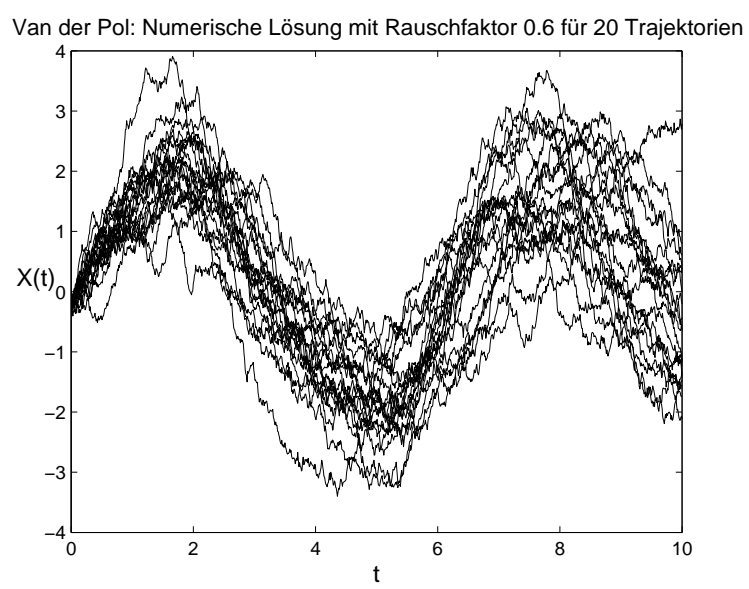

(b)

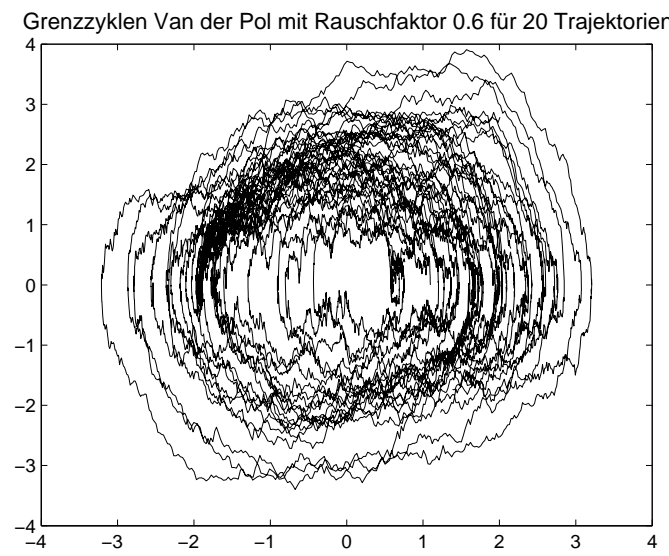

(d)

Abb. 7. Van der Pol Oszillator mit Rauschen. (a) Oszillation mit schwachem Rauschen; (b) Oszillation mit starkem Rauschen; (c) Grenzzyklus mit schwachem Rauschen; (d) Grenzzyklus mit starkem Rauschen.

Die Differentialgleichung des Van der Pol Oszillators wird in dieser Arbeit als Itô-Integral dargestellt und mit Hilfe des Euler-Maruyama Verfahren numerisch gelöst (Kloeden et al., 1994). Zur einfacheren Untersuchung verschiedener Oszillator-Modelle wurde eine graphische Benutzeroberfläche (GUI) (Marchand, 1999) angefertigt (Abb. 6). Durch verändern der Nichtlinearität wird die Form der Oszillation, im Zeitbereich und Zustandsraum dargestellt (siehe Abb. 7), beeinflusst und für $\varepsilon=0.2$ stellt sich ein fast sinusförmiger Verlauf ein. Wie aus Abb. 7c und d zu erkennen ist, wird der Grenzzyklus mit zunehmenden Amplituden- und Phasenrauschen breiter und unförmiger. In den Abb. 8a und b sieht man die Sinusschwingung des Mittelwertes von 50 Trajektorien und die Gaußartigen Wahrscheinlichkeitsdichten zu jedem Zeitpunkt der Oszillation. Die Varianz nimmt mit stärker werdenden Rauschen zu, was sich in der Verbreiterung der Gauß-Kurven deutlich macht. Die Bestimmung der AKF erfolgt mit Hilfe einer zyklischen Erweiterung (siehe Ambardar, 1999) um den Einfluss der Fensterung bei einer endlichen Abtastfolge auszublenden.
In Abb. 9a und b sieht man den AKF-Verlauf der periodisch erweiterten Lösung fr 50 Trajektorien. Im nächsten Schritt wird der Scharmittelwert aller Trajektorien der Autokorrelationsfunktion gebildet, der unverfälschte Teil ausgelesen und der Rand mit Nullen aufgefüllt Abb. $9 \mathrm{c}$ und d.

Der Verlauf der AKF entspricht den analytischen Ergebnissen von Demir, Gleeson und ODoherty (siehe Demir et al., 2000, O'Doherty und Gleeson, 2005), nach denen die AKF eines rauschbehafteten Oszillators, einer Cosinusschwingung mit überlagerter Exponentialfunktion entspricht

$R_{x x}(\tau)=\exp \left(-\frac{1}{2} \Omega^{2} \zeta \tau\right) \cos (\Omega \tau)$.

Die Konstante $\zeta$ in Gl. (20) beschreibt einen Faktor proportional zur Rauschgröße.

Zur Berechnung der SLD wurde das Schätzverfahren nach der Welch-Methode gewählt, da die herkömmliche Periodogramm-Methode schlechtere Ergebnisse liefert (siehe Oppenheim et al., 1989).

Wie zu erkennen ist, hat schwaches Rauschen lediglich Einfluss auf die Amplitude der SLD und nicht auf die Lage ihrer 
(a)

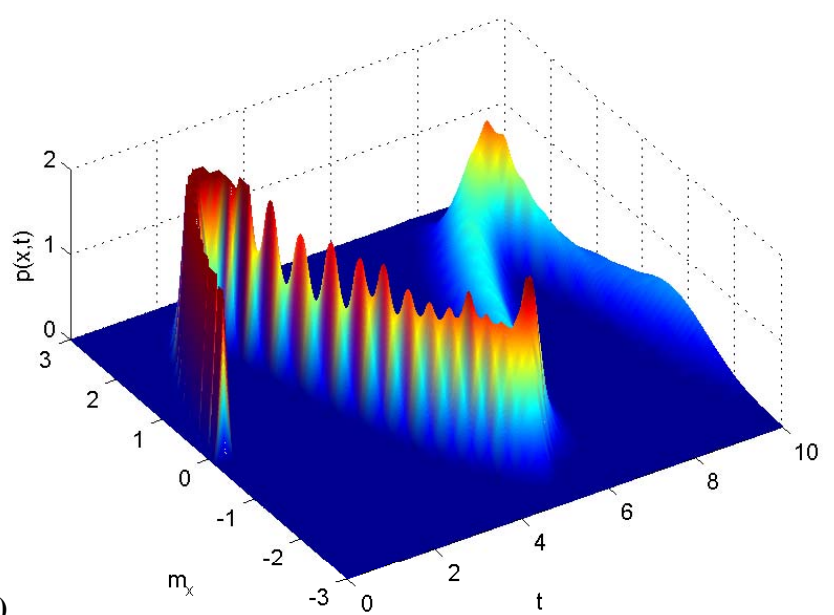

(a)

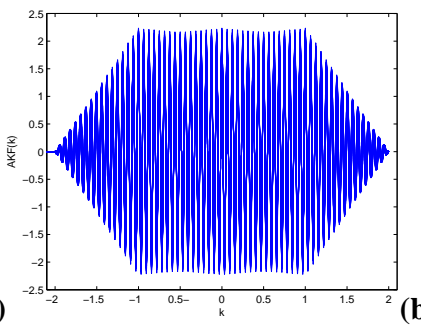

(b)

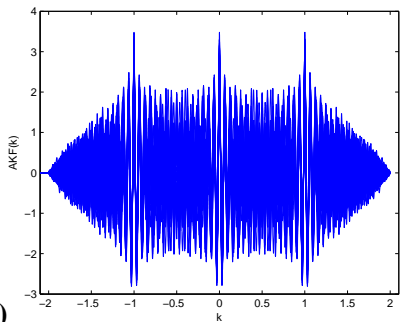

(c)

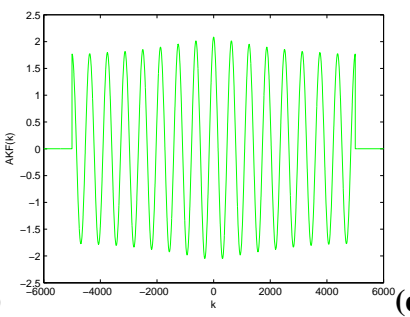

(d)

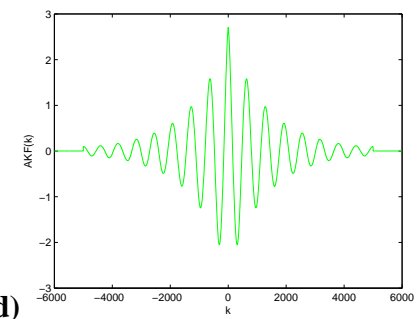

Abb. 9. $\mathrm{AKF}$ von 50 Trajektorien. (a) AKF mit schwachem Rauschen und periodischer Erweiterung; (b) AKF mit starkem Rauschen und periodischer Erweiterung; (c) Gemittelte AKF mit schwachem Rauschen; (d) Gemittelte AKF mit starkem Rauschen.

(a)

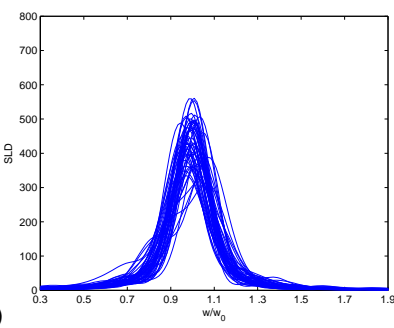

(b)

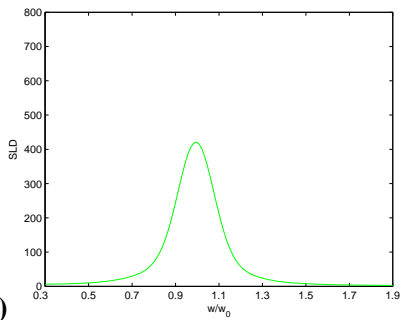

(c)

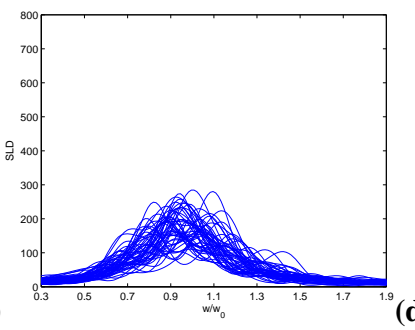

(d)

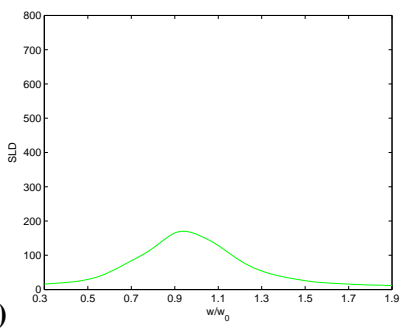

ten Spektrums in Abb. 10b und d. Die SLD einer Oszillation ohne Rauschen müsste die Form einer Delta-Funktion aufweisen. Jedoch entsteht durch das numerische Verfahren eine methoden-bedingte endliche Breite der SLD. Deswegen ist bei der Auswertung die relative und nicht die absolute Breite der SLD relevant.

\section{Diskussion}

In dieser Arbeit wurde erläutert, dass ein Oszillator mit Hilfe eines nichtlinearen Systems modelliert werden muss, welches als Lösungen stabile Grenzzyklen aufweist und somit nach dem Dissipations-Fluktuationstheorem zumindest das thermische Rauschen in Oszillatoren berücksichtigt werden muss.

Abb. 10. Spektrale Leistungsdichte von 50 Trajektorien. (a) SLD mit mittlerem Rauschen; (b) Gemittelte SLD mit mittlerem Rauschen; (c) SLD mit starkem Rauschen; (d) Gemittelte SLD mit starkem Rauschen.

Die in der Praxis verwendeten linearen Modelle wurden genauer untersucht und nachgewiesen, dass sie die spektrale Dispersion rauschender Oszillatoren durch eine lineare Formung des Eingangsrauschens mit Hilfe eines Filterprozesses nachbilden. Die Filterfunktion wird dabei durch eine Impedanzbestimmung ermittelt und erzeugt ein Spektrum in Form einer Gaußartigen Verteilung, die einer Lorentzlinie ähnlich ist. Die in dieser Arbeit verwendeten stochastischen Diffe- 
rentialgleichungen beinhalten dieses Effekt intrinsisch und müssen nicht auf eine Rauschformung zurückgreifen.

Da sich nichtlineare stochastische Differentialgleichungen, analog zu gewöhnlichen Differentialgleichungen, häufig nicht analytisch lösen lassen, wurden sie mit dem numerischen Verfahren nach Euler-Maruyama gelöst. Eine äquivalente Darstellung der stochastischen Differentialgleichung bietet die Fokker-Planck-Gleichung. Ihre Ableitung wurde kurz vorgestellt, wobei jedoch auf ihre Anwendung angesichts der aufwendigen Lösung verzichtet wurde.

Es wurde eine Toolbox in Matlab mit einer graphischen Benutzeroberfläche entwickelt, welche die Eigenschaften, wie Oszillation- und Grenzzyklusverlauf, Wahrscheinlichkeitsdichte, AKF und SLD einer stochastischen Differentialgleichung bestimmen kann. Beispielhaft wurde die numerische Simulation anhand der Van der Pol SDE nach Itô mit Hilfe des Euler-Maruyama Verfahrens durchgeführt und gezeigt, dass die Simulation qualitativ und teilweise quantitativ mit den Ergebnissen in der Literatur übereinstimmt.

\section{Literatur}

Ambardar, A.: Analog and Digital Signal Processing, Brooks/Cole Publishing Company, Second Edition, 1999.

Bogoliubov, N. N. und Mitropolsky, Y. A.: Asymptotic Methods in the Theory of Random Noise, Gordon and Breach, New York, 1963.

Craninckx, J. und Steyaert, M. S. J.: Low-noise voltage-controlled oscillator using enhanced LC-tanks, IEEE T. Circuits-II, 42(12), 794-804, 1995.

Demir, A., Mehrotra, A., und Roychowdhury, J.: Phase noise in oscillators: A unifying theory and numerical methods for characterization, IEEE T. Circuits-I, 47(5), 655-673, 2000.

Hajimiri, A. und Lee, T. H.: A general theory of phase noise in electrical oscillators, IEEE J. Solid-St. Circ., 33, 179-194, 1998.

Hajimiri, A. und Lee, T. H.: Low Noise Oscillators, Kluwer Academic Publishers, 1999.

Hänggi, P. und Riseborough, P.: Dynamics of nonlinear dissipative oscillators, Am. J. Phys., 51(4), 347-352, 1983.

Jordan, D. W. und Smith, P.: Nonlinear Ordinary Differential Equations, Oxford University Press, Second Edition, 1987.

Kärtner, F. X.: Untersuchung des Rauschverhaltens von Oszillatoren, Dissertation an der Technischen Universität München, 1989.
Kloeden, P. E., Platen, E., und Schurz, H.: Numerical Solution of SDE Through Computer Experiments, Springer Verlag, Berlin Heidelberg, 1994.

Kurz, G. und Mathis, W.: Oszillatoren: Schaltungstechnik, Analyse, Eigenschaften, Hüthig Buch Verlag, Heidelberg, 1994.

Lax, M.: Classical Noise, V. Noise in Self-Sustained Oscillators, Phys. Rev., 160(2), 290-306, 1967.

Leeson, D. B.: A Simple Model of Feedback Oscillator Noise Spectrum, P. IEEE, 54, 329-330, 1966.

Maffezzoni, P.: Frequency-Shift Induced by Colored Noise in Nonlinear Oscillators, IEEE T. Circuits-II, 54(10), 887-891, 2007.

Magierowski, K. und Zukotynski, S.: CMOS LC-Oszillator PhaseNoise Analysis Using Nonlinear Models, IEEE T. Circuits-I, 51(4), 664-677, 2004.

Marchand, P.: Graphics and GUIs with MATLAB, CRC Press LLC, Second Edition, 1999.

Mathis, W. und Russer, P.: Oscillator Design, in: K. Chang, Encyclopedia of RF and Microwave Engineering, Volume 4, S.35633589, John Wiley and Sons, 2005.

Mathis, W. und Weiss, L.: Noise Analysis of Nonlinear Electrical Circuits and Devices, in: Modling, Simulation and Optimization of Integrated Circuits, edited by: Antreich, K., Bulirsch, R., Gilg, A., and Rentrop, P., International Series of Numerical Mathematics, 146, 269-282, 2003.

Nallatamby, J., Prigent, M., Camiade, M., und Obregon, J.: Phase noise in oscillators: Leeson formula revisited, IEEE T. Microw. Theory, 51(4), 1386-1394, 2003.

O'Doherty, F. und Gleeson, J. P.: Amplitude-phase coupling effects on the spectral lineshape of oscillators, Proc. SPIE Vol. 5847 Noise in Communications Systems, edited by: Georghiades, C. N. and White, L. B., 31-41, 2005.

Oppenheim, A. V., Schafer, R. W., und Buck, J. R.: Discrete-Time Signal Processing, Prentice-Hall, Second Edition, 1999.

Papoulis, A.: Probability, Random Variables, and Stochastic Processes, McGraw-Hill Book Company, 1965.

Risken, H.: The Fokker-Plack Equation, Springer Verlag, Second Edition, 1989.

Sauvage, G.: Phase noise in oscillators: A mathematical analysis of Leeson's model, IEEE T. Instrum. Meas., IM-26(4), 408-410, 1977.

Stratonovich, R. L.: Topics in the Theory of Random Noise, Gordon and Breach Science Publisher, 1967.

Van Kampen, N. G.: Stochastic Processes in Physics and Chemistry, Elsevier Science Publishers B.V., 1992.

Weiss, L. und Mathis, W.: A thermodynamic noise model for nonlinear resistors, IEEE T. Electron Dev. Lett., 20, 402-404, 1999. 